\title{
ROAp STARS THROUGH THEORISTS' EYES - EXCITATION MECHANISM
}

\author{
ALFRED GAUTSCHY \\ Astronomisches Institut der Universität Basel, Venusstrasse 7, \\ CH-4102, Binningen, Switzerland \\ AND \\ HIDEYUKI SAIO \\ Astronomical Institute, School of Sciences, Tohoku University, \\ Sendai 980-77, Japan
}

\begin{abstract}
This exposition focuses on the still elusive excitation mechanism of the rapid oscillations in some Ap stars. We visit the domain of overstable magnetic convection which was conjectured as the origin of the observed high radial-order $p$ modes. Mostly we dwell, however, on the classical $\kappa$-mechanism. In roAp stars, the zone of partial hydrogen ionization is driving rather effectively. Furthermore, we argue that an atmospheric temperature inversion supports the $\kappa$-mechanism driving by preventing oscillation energy from leaking into the atmosphere.
\end{abstract}

\section{Preliminaries}

Since the discovery of the short-periodic light variation in a cool Ap star (Kurtz 1978), the number of rapidly oscillating Ap (roAp) stars has grown to about 30 . A number of excellent recent reviews addressing various properties of these stars is already available. The most comprehensive article discussing observational as well as theoretical issues of the class of roAp stars was written by Kurtz (1990). Matthews (1991) contributed an observation oriented review emphasizing the aspect of asteroseismology. The most recent observational developments were compiled in the paper of Martinez and Kurtz (1995). Shibahashi's (1991) exposition highlighted theoretical problems remaining with the roAp stars, in particular he contemplated the effects of the magnetic field on the oscillation spectrum. Recently, the perturbation approaches adopting higher-order magnetic multipoles to approximate the stellar field reached new complexities to phenomenologically match the observed oscillation spectra of roAp variables (Shibahashi 1993, Takata \& Shibahashi 1995). We aspire here merely

(C) 1998 IAU. Printed in the Netherlands. 
to complement the before-mentioned expositions and focus therefore on the excitation mechanism of the rapid oscillations. Part of the article reviews the literature, but some new computations and results are also presented.

We begin by summarizing those observed properties which might prove important when considering the excitation mechanism responsible for the roAp stars. They are main-sequence or post - main-sequence stars with $1.5-2 M_{\odot}$. On the HR diagram, all roAp stars seem to reside within the $\delta$ Sct region of the instability strip; i.e. rapid oscillations seem to be excited only in cool Ap stars $\left(6800 \mathrm{~K}<\log T_{\text {eff }}<8400 \mathrm{~K}\right)$. In other words, a clear-cut instability domain seems to exist for the roAp stars. The periods of light variability range from 5 to $15 \mathrm{~min}$ (corresponding to frequencies between 1 and $3 \mathrm{mHz}$ ); this is much shorter than the periods of $\delta$ Sct star oscillations which lie between $30 \mathrm{~min}$ and $6 \mathrm{hr}$. The oscillation periods, together with the integrated-light variability, of roAp stars indicate high-order nonradial $p$ modes of low degree $(\ell=1$ and 2) are excited. Despite the overlap of the roAp-stars instability region with the $\delta$ Sct one, the roAp stars show no $\delta$ Sct - type oscillations. In most cases, only few modes in a small frequency range are simultaneously excited. (A notable exception is HD 60435, in which many modes exist - ranging from $0.7 \mathrm{mHz}$ to $1.5 \mathrm{mHz}$. The lifetimes of some of its modes are shorter than the 7.7 day rotation period [Matthews, Kurtz, Wehlau 1987].) The pulsation amplitudes are modulated with the period of rotation. Kurtz (1982) found the amplitude modulation to be understandable as axisymmetric nonradial oscillations with $\ell=1$ (and 2) whose pulsation axis coincides with the magnetic axis of the star (oblique pulsator model). The strong (dominantly dipolar) magnetic fields encountered in Ap stars are of the order of a kilo-Gauss.

\section{Contemplated excitation mechanisms}

The roAp star excitation mechanisms proposed so far can be divided into two groups: First, resonant driving by overstable magnetic convection and second, classical $\kappa$ mechanisms.

\subsection{OVERSTABLE MAGNETIC CONVECTION MECHANISM}

The idea of overstable magnetic convection as a driving agent for roAp pulsations was proposed by Shibahashi (1983). Its physical meaning was elaborated further by Cox (1984). In a zone with a super-adiabatic temperature gradient, a perturbed blob of matter is pictured to ascend or descend and to develop into 'classical' convection in the absence of a magnetic field (or rotation). If a strong enough magnetic field prevails, however, convective motion is stabilized in the sense that the motion of the blob of matter turns oscillatory with a frequency $\omega_{0}$ given by

$$
\omega_{0}^{2}=N^{2}\left(k_{h}^{2} / k^{2}\right)+(B \cdot k)^{2} /(4 \pi \rho) \text {. }
$$

The square of the Brunt-Väisälä frequency is denoted by $N^{2}$ (which is negative when the temperature gradient is superadiabatic), $k$ is the vectorial wavenumber whereas $k_{h}$ denotes its horizontal component, and $\boldsymbol{B}$ stands for the magnetic induction. The oscillation period $-2 \pi / \omega_{0}-$ is of the order of the thickness of the super-adiabatic zone divided by the Alfvén speed. The heat exchange between the blob and surrounding matter makes the amplitude of the oscillation grow; i.e. an overstable oscillation develops. This behavior is frequently referred to as overstable convection. Under nearly 
adiabatic conditions, the amplitude-growth is proportional to

$$
\exp \left[\frac{1}{2} \nu_{T} k_{h}^{2} \frac{g}{H_{P}} \frac{\chi_{T}}{\chi_{\rho}} \frac{\left(\nabla_{T}-\nabla_{\mathrm{ad}}\right)}{\omega_{0}^{2}} t\right]
$$

where $\nu_{T}$ is the thermal diffusivity, $g$ is gravity, $H_{P}$ the pressure scale-height, $\chi_{T}=$ $(\partial \ln P / \partial \ln T)_{\rho}, \chi_{\rho}=(\partial \ln P / \partial \ln \rho)_{T}, \nabla_{T}=d \ln T / d \ln P$, and $\nabla_{\mathrm{ad}}=(d \ln T / d \ln P)_{\mathrm{ad}}$. Both Shibahashi (1983) and Cox (1984) argued that the periods of overstable convection in a thin super-adiabatic zone in the outer envelope of an Ap star are of the same order as the observed periods of roAp stars. The growth time was estimated to be a few weeks to months (Cox 1984).

For overstable convection to serve as an excitation mechanism for roAp oscillations, we must assume that the resonance between the overstable convective motion and the global high-order $p$ modes works well enough to raise the amplitudes of the $p$ modes to an observable level. A quantitative global analysis to confirm or to deny this conjecture is still lacking. If the resonance hypothesis works indeed, the mechanism implies that only Ap stars with strong magnetic fields show rapid oscillations. If theorists are unable to advance sufficiently rapidly to elaborate on this hypothesis, observers might step in with accurate magnetic-field measurements for an appropriate sample of Ap stars. Shibahashi (1983) and Cox (1984) hypothesized furthermore about why only cool Ap stars show rapid oscillations. Global oscillations can be resonantly excited only for sufficiently thick superadiabatic zones wherein oscillatory convection is also energetic enough to force global modes to observable amplitudes. For the A-type main-sequence stars it was conjectured that this happens where the roAp stars are located.

Whether global modes of low $\ell$ are excited by the above mechanism is far from clear. According to eq. (2), the growth rate is higher for overstable convection with larger horizontal wavenumber. Therefore, we would expect global modes with short horizontal wavelengths to experience strong resonance effects and to be most easily excited. However, only low $\ell$ modes $(\ell=1$ and 2$)$ are detected in roAp stars; this seems to contradict the theoretical prediction, even if the effect of low visibility of high- $\ell$ modes is accounted for (see Dziembowski \& Goode 1996). On the theoretical side, a global analysis is overdue and unavoidable in order to strengthen the case for overstable convection as a driving agent; this is a very difficult, if not impossible task.

\subsection{PARTIAL IONIZATION AND THE $\kappa$ MECHANISM}

The $\kappa$-mechanism takes effect when the stellar radiative energy flow is blocked in the compressed phase and released in the expanded phase of an oscillation. Roughly speaking, this occurs - under weak nonadiabatic conditions - if

$$
\frac{d}{d r}\left(\kappa_{\mathrm{T}}+\frac{\kappa_{\rho}}{\Gamma_{3}-1}\right)>0
$$

where the opacity derivatives $\kappa_{T}$ and $\kappa_{\rho}$ are defined as $\kappa_{T} \equiv(\partial \ln \kappa / \partial \ln T)_{\rho}$ and $\kappa_{\rho} \equiv(\partial \ln \kappa / \partial \ln \rho)_{\mathrm{T}}$, and $\Gamma_{3}-1 \equiv(d \ln T / d \ln \rho)_{\mathrm{ad}}$ (see e.g. Unno et al. 1989). In the stellar envelope, $\kappa_{\rho}$ is nearly constant and of the order of unity while the variation of $\kappa_{\mathrm{T}}$ plays the essential rôle in the $\kappa$-mechanism. The value of $\kappa_{\mathrm{T}}$ increases outward close to an opacity peak; this is where the $\kappa$-mechanism is most effective (outward refers to the direction of increasing radius in the star). Opacity peaks are usually 


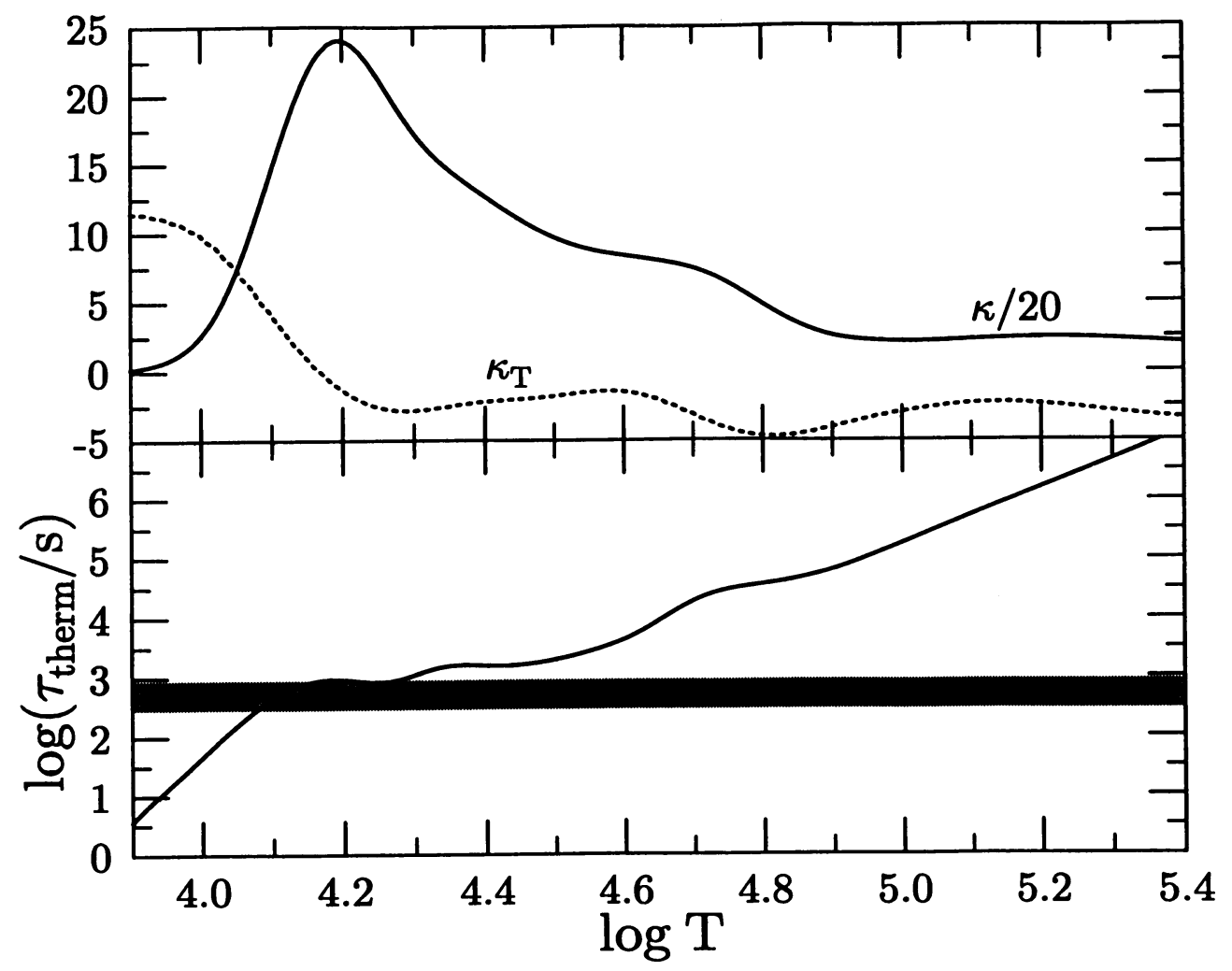

Figure 1. Run of the opacity $\kappa, \kappa_{T}$, and the thermal timescale in a $1.5 M_{\odot}$ ZAMS model with respect to temperature. The grey bar indicates the observed period range of roAp stars.

associated with ionization zones where in the inner part of the peak $\Gamma_{3}$ tends to decrease outward. This behavior of the adiabatic exponent helps to further satisfy inequality (3).

Driving through the $\kappa$-mechanism in an ionization zone is most effective when the thermal timescale $\tau_{\text {therm }}$ of the stellar envelope above that ionization zone is comparable with the pulsation period (Cox 1974). The thermal timescale of a $1.5 M_{\odot} \mathrm{ZAMS}$ model is shown in Fig. 1 as a function of temperature. The $\tau_{\text {therm }}$ was approximated as $\left(M_{*}-M_{r}\right) C_{\mathrm{p}} T / L_{r}$. As seen in Fig. 1, the thermal timescale at the HeII ionization zone of an A-type main-sequence star is of order $10^{4} \mathrm{~s}$ which corresponds to the periods of $\delta$ Sct - type variability. The $\kappa$-mechanism at the HeII ionization zone is indeed the dominant excitation agent of the $\delta$ Sct pulsations. On the other hand, the thermal timescale at the $\mathrm{H}$ (and HeI) ionization zone lies between a few hundred and about $10^{3}$ seconds which is comparable with oscillation periods of the roAp stars (which is marked with the grey band in Fig. 1). This indicates that the $\kappa$-mechanism at the H-ionization zone can potentially excite the short-period modes seen in the roAp stars.

At least two problems have to be overcome before the $\kappa$-mechanism can be re- 


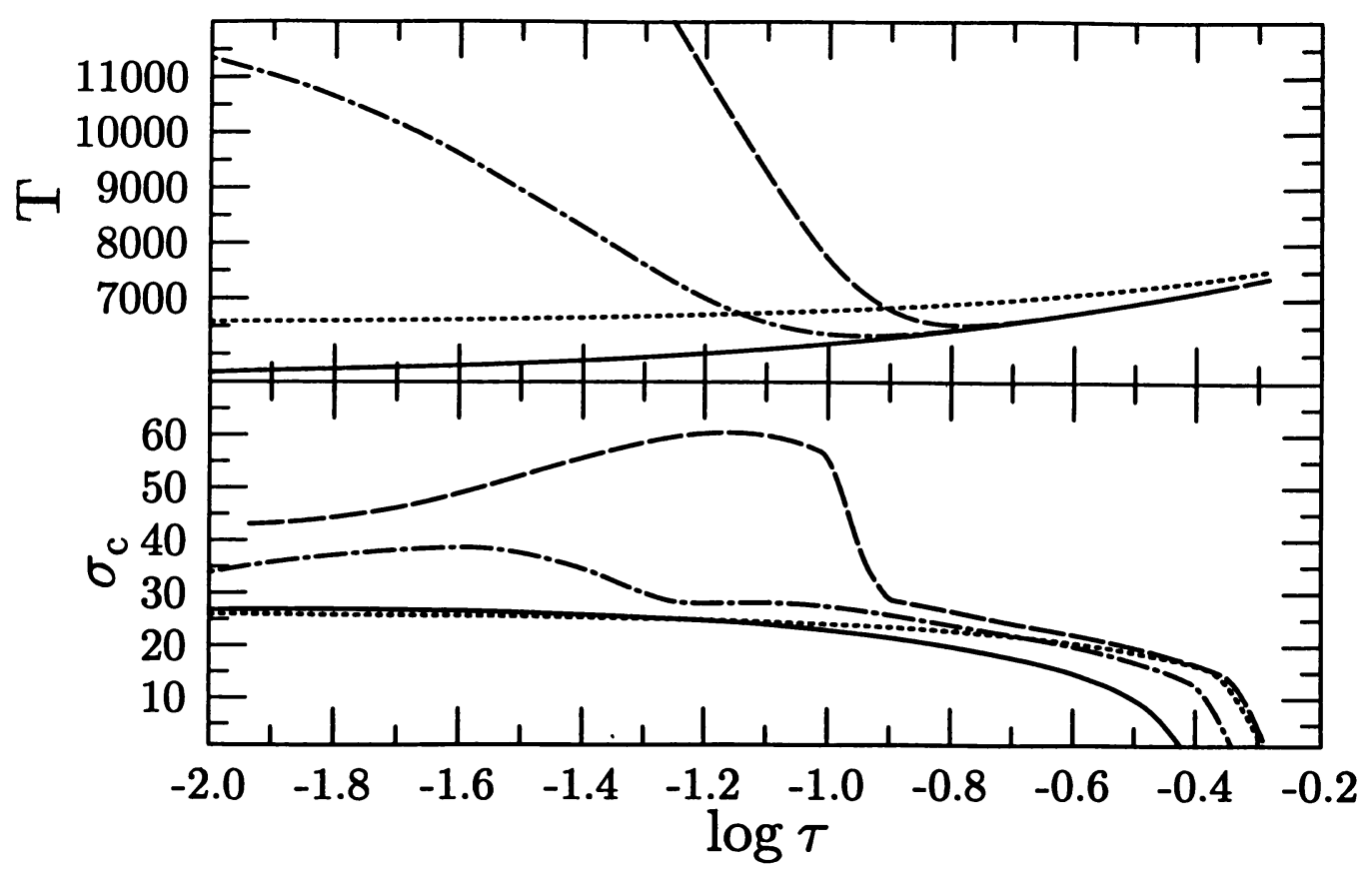

Figure 2. Assumed temperature distributions (upper panel) and corresponding spatial variation of the critical frequency. The horizontal axis represents the logarithm of the optical depth $\tau$. In this normalization, $\sigma_{c} \simeq 30$, for example, corresponds to $2 \mathrm{mHz}$.

garded as the excitation mechanism for roAp-star variability. One problem is that the $\delta$ Sct - type pulsations must be suppressed in roAp stars. Usually, helium is assumed to be drained due to gravitational settling from regions where otherwise partial HeII ionization contributes to pulsational driving. This reasoning works also to argue for the absence of $\delta$ Sct - type oscillations in Am stars whose peculiar spectra are explained by elemental diffusion. It is not certain, however, whether gravitational settling is efficient enough to drain $\mathrm{He}$ as deep as $T \sim 5 \times 10^{4} \mathrm{~K}$, in particular in non-static envelopes. To be candid, it is even controversial whether $\mathrm{He}$ is depleted or enhanced by diffusion. Dolez, Gough, \& Vauclair (1988) argued that He should be increased at the magnetic poles if a stellar wind is included in the full picture. The other problem to be resolved has to do with the critical frequency. In an Eddingtontype atmosphere the critical frequency is lower than the typical roAp-star oscillation frequencies (Shibahashi \& Saio 1985). Despite some uncertainty in the definition of the critical frequency it is clear that the higher the oscillation frequency lies above the critical frequency, the higher the mode-energy leakage at the surface.

Dziembowski \& Goode (1996) examined the pulsational stability for $1.8 M_{\odot}$ and $2.0 M_{\odot}$ models which have almost the same effective temperature of about $8.4 \times 10^{3} \mathrm{~K}$. They noted that in the $2.0 M_{\odot}$ model the driving at the $\mathrm{H}$ ionization zone almost excites high order $\boldsymbol{p}$ modes (i.e., the driving is exceeded only slightly by the radiative damping), and that in the $1.8 M_{\odot}$ model the maximum driving occurs for frequencies above the critical frequency. Their result suggests that if some physical effect can 
be thought of to increase the critical frequency in roAp stars, rapid oscillations are possibly excited by the $\kappa$-mechanism in the partial hydrogen-ionization zone. Such an effect must be attributable to Ap-star peculiarities because no rapid oscillation is detected in $\delta$ Sct or Am stars. One obvious property of Ap stars is the strong magnetic field. The direct effect of a magnetic field is, however, to damp oscillations (Roberts \& Soward 1983; Dziembowski \& Goode 1996). Alfvénic waves are generated in a thin surface layer by coupling $p$-mode oscillations with the magnetic field. As they propagate inward these Alfvénic waves are expected to be dissipated because their wavelength becomes very small.

\section{Temperature inversion}

A temperature inversion, as it prevails in the solar chromosphere, is not unconceivable in Ap stars due to the presence of strong magnetic fields and a superficial convective region due to the partial $\mathrm{H}, \mathrm{HeI}$ ionization zone. We note that a temperature inversion in the atmosphere can increase the critical frequency significantly. The adiabatic critical frequency $\omega_{c}$ for high order $p$ modes may be written as

$$
\omega_{c}^{2} \simeq \frac{G M}{R^{3}} \frac{\Gamma_{1}}{2}\left(\frac{d \ln \rho}{d \ln r}-\frac{V}{2}\right)=\frac{G M}{R^{3}} \frac{\Gamma_{1} V}{2}\left(\frac{1}{\chi_{\rho}}-\frac{\chi_{T}}{\chi_{\rho}} \nabla_{T}-\frac{1}{2}\right)
$$

(cf. Shibahashi \& Saio 1985), where $V=-d \ln P / d \ln r$. As seen in eq. (4), a negative $\nabla_{T}$ increases the critical frequency for adiabatic $p$ modes. (Since high-order $p$ modes as seen in roAp stars should be quite nonadiabatic, the exact critical frequency is, however, somewhat uncertain.)

Figure 2 shows the spatial variations of $\sigma_{c}$ (which corresponds to $\omega_{c}$ normalized by $\sqrt{3 G M / R^{3}}$ ) for some examples of the $T-\tau$ relation with and without temperature inversion. Clearly a temperature inversion in the atmosphere can sufficiently increase the critical frequency to suppress atmospheric energy leakage of roAp-type shortperiod oscillations. Actually, we have confirmed that in a chemically homogeneous model with a temperature inversion, roAp type oscillations as well as $\delta$ Sct - type oscillations are overstable. The temperature inversions used for these computations are rather strong and would possibly leave observable chromospheric evidence which was not seen as reported by Shore et al. (1987). We notice, however, that if the atmospheric temperature inversion is produced only by a strong magnetic field in A-type stars, we do expect no temperature inversion and hence no short period oscillations in Am or $\delta$ Sct stars. Therefore, a temperature inversion is, after all, a rather elegant concept to increase the cut-off frequency, so that we currently follow up this avenue also with weaker ones.

Assuming slight gravitational settling of helium in the outermost part of the envelope we found $\delta$ Sct - type pulsations to be indeed suppressed. One example is shown in Fig. 3, where the imaginary part (appropriately scaled: $\Sigma_{I}=\operatorname{sign}\left(\sigma_{I}\right) \cdot \log (1+$ $\left.\left|\sigma_{I}\right| / 10^{-4}\right)$ ) of the eigenfrequency is plotted as a function of period for a population $I$ model with helium depleted by 0.1 (in mass fraction) in the regions with $\log T<4.7$. The lack of excited long-period oscillations occurs even for a slight decrease of helium because in such an inhomogeneous envelope the hydrogen ionization zone acts as a damping zone. This result suggests that helium need not to be drained completely from the HeII ionization zone to suppress the $\delta$ Sct - like oscillations.

To obtain a definite answer on the excitation mechanism of roAp oscillations, the effect of magnetic fields must be included in the stability analysis. The magnetic pres- 


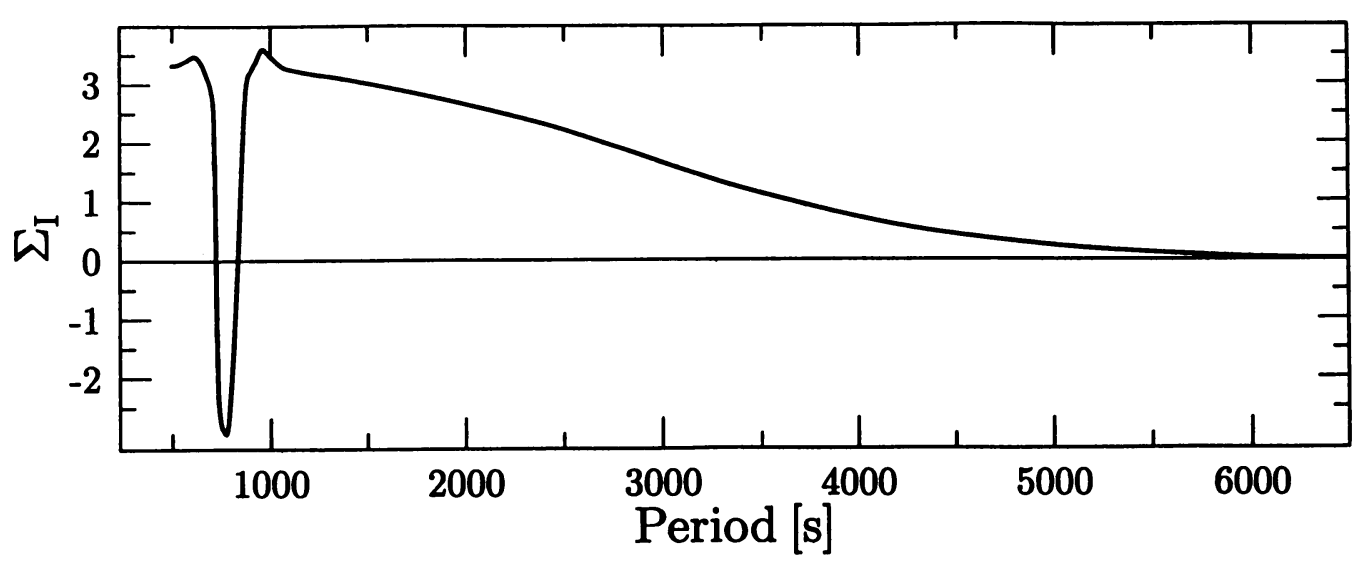

Figure 3. Appropriately scaled imaginary parts $\Sigma_{I}$ of the eigenfrequency versus period for a model with a inhomogeneous envelope. A negative $\Sigma_{I}$ indicates an overstable the mode.

sure of a kilo-Gauss magnetic field is much higher than the gas pressure only in the outermost zone. Therefore, the magnetic effect on the oscillation frequency is expected to be very small, but the effect on the stability might be considerable. Solving linear pulsation equations including magnetic effects in the adiabatic approximation, Dziembowski \& Goode (1996) showed that the damping effect of magnetic fields can reach the magnitude of radiative damping. A self-consistent, fully nonadiabatic analysis is, however, needed. This is very demanding because the magnetic field requires that the latitudinal dependence of an eigenfunction for a given $m$ can no longer be represented by a single associated Legendre function $P_{\ell}^{m}(\cos \theta)$. The importance of each $\ell$ component changes as the strength of magnetic field changes. An additional complexity enters through avoided crossings, i.e. mode interactions, which occur between modes having different latitudinal dependencies.

\section{Conclusion}

If Ap stars can develop a temperature inversion in their atmospheres, and if a slight helium settling obtains in their envelopes, the problem of exciting rapid oscillations in some of them could be solved. Before a definite answer is obtained in this direction, however, a self-consistent, fully nonadiabatic analysis including the effect of magnetic fields is necessary.

Acknowledgments: A.G. was financially supported by the Swiss National Science Foundation through a PROFIL2 fellowship. H.S. acknowledges financial support from the Swiss National Science Foundation and the Japan Society for the Promotion of Science, which enabled him to stay and work on this project at the Astronomisches Institut der Universität Basel. 


\section{References}

Cox, J.P. 1974, Rep. Prog. Phys., 37, 563

Cox, J.P. 1984, ApJ, 280, 220

Dolez, N., Gough, D.O., Vauclair, S. 1988, in 'Advances in Helio- and Asteroseismology', IAU Symp. No. 123, eds. J. Christensen-Dalsgaard and S. Frandsen, (Reidel, Dordrecht), p. 291

Dziembowski, W.A., Goode, P.R. 1996, ApJ, 458, 338

Kurtz, D.W. 1978, IBVS, No 1436

Kurtz, D.W. 1982, MNRAS, 200, 807

Kurtz, D.W. 1990, ARA\&A, 28, 607

Martinez, P., Kurtz, D.W. 1995, in 'Astrophysical Applications of Stellar Pulsation', eds. R.S., Stobie and P.A. Whitelock, ASP Conference Series, Vol. 83, 58

Matthews, J.M. 1991, PASP, 103, 5

Matthews, J.M., Kurtz, D.W., Wehlau, W. 1987, ApJ, 313, 782

Roberts, P.H., Soward, A.M. 1983, MNRAS, 205, 1171

Shibahashi, H. 1983, ApJ, 125, L5

Shibahashi, H. 1991, in 'Challenges to theories of the structure of moderate-mass stars', eds. Gough, D.O. \& Toomre, J., (Springer, Berlin) p. 393

Shibahashi, H. 1993, in 'GONG'94: Helio- and Asteroseismology', eds. R.K. Ulrich, E.J. Rhodes Jr. and W. Däppen, ASP Conference Series, Vol. 76, p. 618

Shibahashi, H., Saio, H. 1985, PASJ, 37, 245

Shore, S.N., Brown, D.N., Sonneborn, G., Gibson, D.M. 1987, A\&A, 182, 285

Takata, M., Shibahashi, H. 1995, PASJ, 47, 219

Unno, W., Osaki, Y., Ando, H., Saio, H., Shibahashi, H. 1989, 'Nonradial Oscillations of Stars' (University of Tokyo press) 